\title{
Dissecting the Heterogeneity of Breast Cancer*
}

\author{
Peter Dubsky Raimund Jakesz \\ Universitätsklinik für Chirurgie, Medizinische Universität Wien, Austria
}

\section{Key Words}

Breast cancer biology · Genetics · Angiogenesis . Inflammation · Immunity · Hormone receptors

\section{Summary}

Breast cancer is a highly heterogeneous disease with respect to its biology. In recent years, clinical and basic research have delivered data concerning tumor biology stemming from multiple methodological backgrounds, which in many cases are highly complementary to our understanding of breast cancer. For example, genetic profiling has identified subtypes of breast cancer that are both largely overlapping and complementary to data gathered in clinical research. In endocrine-related breast cancer, we can now not only formulate fairly good prediction, we can also link this knowledge to the molecular background of a tumor subtype. The following review attempts to summarize novel insights into breast cancer tumor biology as presented by representatives of clinical and basic research during the 2007 St. Gallen Consensus Meeting.

\section{Background}

The past decades of oncology research have revealed breast cancer to be a highly heterogeneous disease. The early discovery of endocrine-dependent growth of breast tumors, as op-

"Presentations at the 10th International Conference on 'Primary Therapy of Early Breast Cancer', St. Gallen, Switzerland, 2007

\section{Schlüsselwörter}

Biologie des Mammakarzinoms · Genetik · Angiogenese · Entzündung · Immunität · Hormonrezeptoren

\section{Zusammenfassung}

Brustkrebs ist bezüglich seiner Biologie eine uneinheitliche Erkrankung. In den letzten Jahren haben klinische und basiswissenschaftliche Forschungsansätze mit zum Teil völlig unterschiedlicher Methodik sehr gut miteinander verknüpfbare Erkenntnisse erbracht. Beispielsweise hat die Unterteilung des Mammakarzinoms in genetische Subtypen eine zur klinischen Forschung weitgehend komplementäre Einteilung festgelegt. Im Bereich der endokrin abhängigen Mammakarzinome können wir nun nicht nur eine recht genaue Prädiktion durchführen, wir haben auch eine Verknüpfung zu den molekularbiologischen Ursachen erstellt. Die vorliegende Übersichtsarbeit versucht eine Zusammenfassung neuer tumorbiologischer Erkenntnisse zu erstellen, welche durch Vertreter der klinischen und basiswissenschaftlichen Forschung im Rahmen des Konsensus-Meetings in St. Gallen 2007 vorgetragen wurden. posed to the possibility of cancer progression in the absence of endocrine receptors, was one of the pivotal initial findings of breast cancer biology. The appeal of such biological studies was evident, since endocrine receptors did not only correlate closely with prognosis, but were able to predict response to antihormonal therapy. Using fundamental biological differences as such, an intellectual dissection of breast cancer into disease subtypes has broadened the field unlike any other solid organ cancer and has gradually altered our daily medical

\begin{tabular}{ll}
\hline KARGER & @ 2007 S. Karger GmbH, Freiburg \\
Fax +497614520714 & Accessible online at: \\
$\begin{array}{l}\text { E-mail Information@Karger.de } \\
\text { www.karger.com }\end{array}$ & www.karger.com/brc \\
&
\end{tabular}


practice. During the St. Gallen oncology conferences on the primary therapy of early breast cancer, two sessions reviewed some of the recent tumor biology advances in the field. Furthermore, these reviews shed light on some of the current and future implications on breast cancer therapy.

The following review will categorize the research presented at the meeting according to the biological backgrounds that break down breast cancer into subtypes:

1. Immunity and inflammation: understanding host response to breast cancers and finding potential therapeutic targets (Giuseppe Curigliano, Alberto Mantovani; Italy)

2. Genetics: genetic profiling and correlating biologic traits dissect breast cancers and open new avenues for prognosis and prediction (Charles Perou, Judy Garber; USA)

3. Steroid hormone receptors: polymorphism of endocrine response and crosstalk with other growth factors (Paolo Ciana, Italy; Kent Osborne, USA)

4. Angiogenesis: a target in breast cancer (Dan Hayes, USA)

5. Self-seeding of cancer cells: integrating cell movement into the understanding of cancer growth (Larry Norton, USA).

\section{Immunity and Inflammation}

The definition of tumor antigens recognized by immune effector cells has raised hopes of developing breast cancer vaccines. Indeed, the promise of nontoxic immune responses against cancer both make combination therapies possible and suggest that immunological memory may prevent the reemergence of cancer. These hopes are certainly justified in that minimal residual disease could be targeted due to a particular antigenic profile of few remaining cancerous cells.

Cancer vaccines have been derived from tumor cells (irradiated or transfected) and tumor lysates [1]. Examples of breast cancer immunotherapy also include vaccines based on dendritic cells [2] and DNA/RNA that encodes for tumor antigens. Dr. Curigliano (European Institute of Oncology, Division of Medical Oncology, Milano, Italy) focused the subject of breast cancer vaccines on recent advances made using a truncated, recombinant Her-2 protein (dHer2) in combination with a potent immunological adjuvant (AS15). Data from phase I trials demonstrate safety and the absence of severe side effects during treatment. Interestingly, the vaccine induces antibody responses to both the extracellular and intracellular domain of Her-2/neu. Antibodies generated can bind to the Her-2/neu receptor, and specific CD4- and CD8-T cell responses can be measured. Genetic signatures similar to those observed after trastuzumab treatment have been seen in vaccinated patients.

Several examples in recent literature point to tumor growth via inflammatory reactions. From the epidemiologic point of view, several cancers such as bladder, colon, gastric, and cervical cancer among many have been associated with inflammation. Genetic profiling of breast cancer has identified immune response signatures in at least a subtype of the disease [3]. Notably, CD68, a defining cluster of differentiation for macrophages, has been incorporated in a validated predictive signature for recurrence in tamoxifen-treated patients [4]. Dr. Mantovani (Istituto Clinico Humanitas, Milano, Italy) was able to make a convincing connection between breast cancer and inflammation during the introduction of his talk.

Specifically, many types of breast cancer display inflammatory infiltration by macrophages (tumor-associated macrophages, TAMs), a phenomenon that has been correlated with poor outcome. Recent evidence suggests that the peritumoral inflammatory reaction and VEGF-C expressing TAMs may play an important role in tumor lymphangiogenesis and lymphovascular invasion in invasive breast cancer [5].

TAMs are suggested to display the functions of what has been described as an M2 polarization of macrophages [6]. This polarization of macrophages is associated with tissue remodeling and repair in addition to chronic inflammatory response seen in established invasive cancer.

An interesting example of how oncogenetic defects can create an intrinsic inflammatory response of cancer cells that can even be potentially causative for neoplastic transformation has recently been demonstrated for papillary thyroid cancer (PTC) [7]. The rearrangement of the RET/PTC oncogene in human thyrocytes has been shown to activate a proinflammatory program including several chemokines and chemokine receptors. As reported by Dr. Mantovani during the meeting, the pattern of these molecules is also expressed in invasive primary, and even more so in metastatic tumors. These results link genetic events that are causative for cancer directly to inflammation.

Certainly, classic extrinsic pathways to inflammation can be targeted and have shown success in clinical prevention trials: human papilloma virus (HPV) and cervical cancer, hepatitis B virus $(\mathrm{HBV})$ and hepatoma. Intrinsic pathways as described above may provide future therapeutic targets.

\section{Genetics}

In a review of patients with genetic susceptibility to breast cancer, Judy Garber (Dana Farber Cancer Institute, Boston, USA) presented several examples on how the study of very few individuals with hereditary risk can give clues to underlying biological mechanisms that are applicable to several subsets of sporadic breast cancer. Germline mutations in BRCA1/2 and Fanconi anemia genes are responsible for disrupted DNA break repair [8]. Several lines of data indicate that similar mechanisms occur in sporadic cancer. This 'BRCAness' [9] of a subset of sporadic breast cancer is pertinent to their clinical management.

The most important example in this respect is the study of BRCA1 mutation carriers. Phenotypic studies of the basal-like breast cancer subtype (outlined below) have shown large con- 
cordance with tumors from BRCA1-deficient patients: $80 \%$ of mutation carriers show a triple-negative (estrogen/progesterone receptor-negative, Her-2/neu-negative) phenotype. There is a low expression of cyclin E, p27, and AKT, while CK 5/6 and CK17 are often found in immunohistochemistry. Very much like the basal-like subtype, there is a high rate of p53 mutation, and the amplification of c-myc and epidermal growth factor receptor (EGFR) is common.

Despite the fact that BRCA1 is intact in sporadically occurring basal-like tumors as defined across several microarray platforms, there seem to be several underlying biological similarities between the BRCA1-mutated tumors and the intrinsic basal-like subtype. The strongest line of evidence to support this notion comes from genetic profiling of hereditary breast cancer and the basal-like subtype of breast cancer. The two types of tumor segregate with each other and thus indicate a common etiology of neoplasia. The common study of these subtypes has already led to the identification of novel therapeutic targets such as c-KIT and EGFR. Furthermore, cell line data suggest good response to platinum-containing regimens and several novel biological agents such as poly(ADP-ribose) polymerase-1 (PARP-1) inhibitors and tyrosine kinase inhibitors. Several clinical trials, mostly in the preoperative setting, are under way.

In summary, the study of hereditary breast cancer has identified germline mutations in at least 10 different genes, active in pathways to genomic integrity. The combined role of these genes reveals some of the underlying biology of a tumor and pinpoints novel targets. The parallel findings of sporadic breast cancer genetics by high output technologies such as microarray analysis may make biochemical pathways identified in a minuscule subset of mutated patients applicable to a larger group of sporadic breast cancers.

Microarray analysis has emerged as the major tool to genetically profile breast cancer. Although several predictor tools based on genetic expression have been developed and some have shown good clinical concordance [10], only two have reached the stage of testing in a prospective clinical trial.

Charles Perou, PhD from the University of North Carolina, reviewed the development of gene pattern expression that identified breast cancer subtypes with high prognostic significance. Five breast cancer subtypes originating from at least two distinct cells (epithelial-like and basal-like cells) were identified [11] (intrinsic/Stanford gene list). After further development of these original gene lists (intrinsic/UNC gene list), the data has recently been applied to a combined test set. Independent data from three researchers (and four microarray platforms) was pooled into a single gene list using complex statistical procedures. Then hierarchical clustering analysis was performed on a total of 315 samples and using 306 genes. In this analysis, several genes were lost, due to the requirement of having to be present across all four platforms. Nevertheless, the five breast cancer subtypes (luminal A and B, Her2+/ER-, basal-like, and normal breast-like) were identi- fied and corresponded to the previous data. Having validated the subtyping of breast cancer, the researchers set out to determine the predictive value of the intrinsic/UNC gene set. A multivariate model containing standard clinical variables (such as age, node status, tumor size etc.) and the five subsets was created. Genetic profiling was indeed shown to add prognostic information to standard clinical predictors.

The major restriction to hierarchical cluster analysis is its applicability to the individual patient. Classification of each sample would require a total analysis of all patients in the data set. To address the issue of single sample prediction (SSP), a mean expression for each of the subtypes (i.e. centroid) was created. In order to classify the patient, a comparison with each centroid is carried out, and the closest match is determined by Spearman correlation. The SSP tool thus consists of idealized profiles with a significant value in multivariate analysis (LumA, LumB, basal-like, Her+/ER-) to which any new sample can be compared. To validate SSP, two independent data sets and a 105 sample dataset used to derive the intrinsic/UNC gene list were used. SSP correlates with standard clinical parameters and successfully identified clinically relevant groups (e.g. LumA vs. LumB) which otherwise could not have been dissected by using standard clinical markers.

Furthermore, DNA or RNA isolation for microarray analysis at this point is largely restricted to fresh frozen tissue, which at least in daily practice does represent a major impediment. Dr. Perou presented data from Ellis et al. (Washington University) and Bernard et al. (University of Utah) to overcome this problem. A list of 60 genes was generated from frozen tissues using quantitative PCR (qPCR) that allows the identification of subtypes by hierarchical cluster analysis. In a next step, it is now possible to isolate RNA from formalin-fixed, paraffinembedded (FFPE) tissue and perform centroid-based SSP.

In summary, the identification of breast cancer subtypes by hierarchical cluster analysis of microarray data has shown prognostic significance in multivariate analyses on several sets of data. Together with other prediction platforms such as the Oncotype $\mathrm{DX}^{\mathrm{TM}}$ or Mammaprint ${ }^{\mathrm{TM}}$, these intrinsic subtypes are currently under investigation and will serve as new tools in the diagnosis of cancer. They may well be able to predict outcome from treatment and could ultimately define treatment choices.

\section{Steroid Hormone Receptors}

Estrogen signaling is a complex process involving the two nuclear receptors estrogen receptor $\alpha(\mathrm{ER} \alpha)$ and $\mathrm{ER} \beta$ derived from two different genes. These receptors reside in the nucleus and bind to estrogen-related elements on DNA functioning as transcription factors (fig. 1). After the cognate interaction between estrogens and $\mathrm{ER} \alpha$, dimerization occurs, leading to full transcription of genes including those necessary for proliferation. In the past decades, several layers of complexity have been added to this model of signaling, many of which are high- 


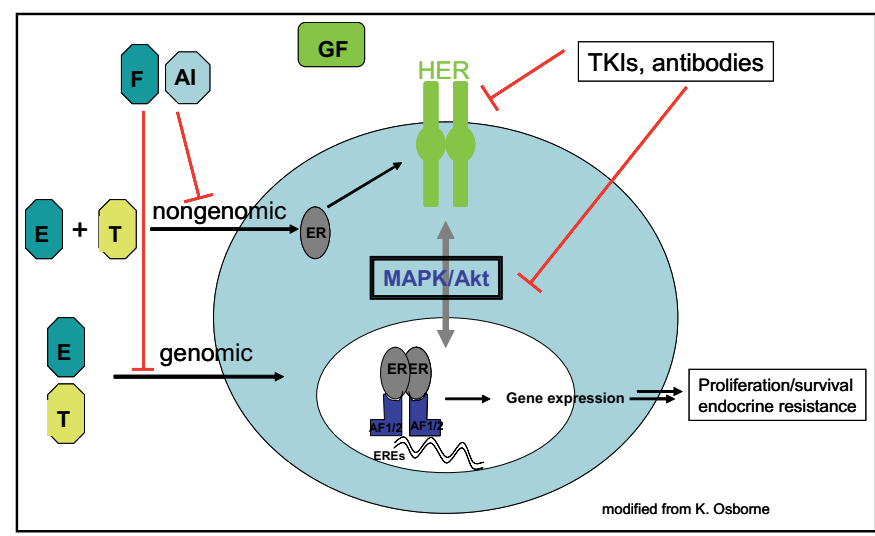

Fig. 1. Estrogen signaling. Estrogen (and tamoxifen) can stimulate proliferation and survival via both genomic and nongenomic functions. Aromatase inhibition or downregulation of ER in the nucleus and the cytoplasm may be able to stop both types of signaling. Inhibition of receptors from the Her family via e.g. tyrosine kinase inhibition or use of antibodies may serve as a further means of overcoming endocrine resistance. $\mathrm{E}=$ Estrogens; $\mathrm{T}$ = tamoxifen; $\mathrm{AI}=$ aromatase inhibitor; $\mathrm{F}=$ fulvestrant $\mathrm{ER}=$ estrogen receptor; AF1/2 = activating function _; HER $=$ Her family receptor; TKI = tyrosine kinase inhibitor; GF = growth factor; EREs = estrogen responsive elements; $\mathrm{MAPK}=$ mitogen-activated protein kinase.

ly relevant to the understanding of malignant transformation, growth, metastasis, and response or resistance to therapy.

Transcriptional modulation of estrogen target genes can emerge as a result of interplay between co-activator and -repressors which, according to their existence in the tissue, modulate estrogen action to the specific needs. As a result, selective estrogen receptor modulators (SERMs), substances that induce a less efficient but yet persistent transcription of estrogen-dependent genes, may have a proliferative effect on one tissue and provide inhibitory signals to the next.

A growing body of evidence adds yet another layer to the complexity of ER signaling. Although the majority of ER is found in the nucleus, a small amount of ER is also found in the cytoplasm and the cell membrane (fig. 1). Membrane-associated ER can modulate cell function within minutes of estrogen binding and thus functions without transcriptional activity. The membrane-associated pathways are often referred to as 'nongenomic' functions. Membrane ER can activate a variety of growth factor-signaling molecules, the most prominent being IGF1-R (insulin growth factor 1 receptor), PI3K (phosphatidyl-inositol-3 kinase), IRS1 (insulin receptor substrate) or Shc and Src. The kinase cascade activated via this pathway in turn will lead to ERK1/2- and mitogen-activated protein kinase (MAPK) and Akt activation. Through this pathway, potent survival and proliferative signals are provided without cognate ligation to the ER in the nucleus. Nongenomic ER effects may be of pivotal importance when growth factor signaling is abundant. This is most likely true for breast cancers overexpressing EGFR or Her-2/neu.

Dr. Ciana (Department of Pharmacological Sciences, University of Milan, Italy) pointed out that each pharmacological ER ligand could have activating and repressing effects depending on the target tissue and might also influence a cancer cell as a function of its (epi)genetic background. In order to study the effect of estrogen-related transcription in vivo, the ERE-luc mouse model was engineered. A luciferase reporter gene under control of transcriptionally activated ER is inserted into the mouse genome [12]. The transgenic animal reacts with the synthesis of luciferase proportional to ER activation, which can be monitored in vivo under physiological conditions or under pharmacological treatment. As pointed out during the meeting, this reporter mouse can in the future be designed to reveal other aspects of ER signaling such as the interactions with other nuclear or cytoplasmatic proteins in vivo. One of the main findings reported during the review was the importance of IGF-1 signaling occurring in nonreproductive organs in the absence of estradiol. ER activation in e.g. bone, liver or muscle can occur in the absence of its cognate ligands. Given the association of IGF-1 signaling in human malignancy, it is thus important to understand how, under physiological conditions, reproductive organs can defer IGF-1 action in hormonal phases without high estradiol levels (diestrus phase).

$\mathrm{ER} \alpha$ expression is tightly regulated in reproductive organs. A block to transcript elongation occurs under physiological conditions, which shuts down the growth factor-induced activation of ER $\alpha$. Interestingly, the block in transcription can be modulated by polymorphisms in intron 1 of $E R \alpha$ that have been associated to endometrial and breast cancer (PvuII and Xbal).

Building on an intimate biological understanding of crosstalk between ER and growth factor receptors, Dr. Kent Osborne (Baylor College of Medicine, Houston, Texas, USA) described several strategies to elude endocrine resistance. In tumors that display both genomic and nongenomic effects of ER, hormone deprivation by aromatase inhibitors or the downregulation of ER (fulvestrant) could be strategies that overcome resistance to the selective modulation of ER by e.g. tamoxifen. Otherwise, several new molecules that inhibit growth factor signaling (tyrosine kinase inhibitors) may restore the antagonist function of tamoxifen via its genomic effects. Furthermore, data showing the effectiveness of combination drug therapy that block all HER dimerization (hetero- or homodimers) in xenograft mouse models has been reported.

In summary, the data provided shows a high redundancy of pathways in which ER pathways and growth factor pathways interact with each other. Drugs that initiate multiple specific blocks to growth factors without causing toxicity in combination with endocrine therapy might provide the best antitumor effect in the future.

\section{Angiogenesis}

Over 35 years ago, Judah Folkman proposed that the formation and perpetuation of cancer masses relies on a supply of 
blood. The interaction between cancer cells and stroma that would induce the formation of blood vessels was termed neoangiogenesis. The field of angiogenesis has broadened considerably, and approx. 20 factors that promote or inhibit neoplasia-associated blood vessel formation have been described. As pointed out by Dr. Dan Hayes (University of Michigan Comprehensive Cancer Center, Ann Arbor, USA) during his review, the high expectations about antiangiogenic therapy have recently gained new support. In first-line treatment of metastatic breast cancer, the addition of bevacizumab, a humanized monoclonal antibody targeting vascular endothelial growth factor (VEGF), to taxane-based chemotherapy has shown an increase in objective response rates and time to progression [13]. Side effects of the antibody seem to be fairly well manageable (hypertension), but the overall cost per patient and year is currently calculated to be around 100,000 US\$.

Several attempts of correlative science projects have been made to understand the mechanisms of action associated with response. Currently, the evaluation of circulating angiogenic factors such as VEGF and VCAM-1 has not been able to identify responding or nonresponding patients. The investigation of tissue angiogenesis assays and tissue 'angiogenic profiles' is under way. Furthermore, single nucleotide polymorphisms (SNPs) in VEGF and its receptors may help to identify surrogate markers of response. Considering costs and current therapeutic index, the identification of therapy targets will be critical to the implementation of bevacizumab.

As tumor size increases, the angiogenic pathways it can access broaden considerably and redundancy occurs. The implementation of anti-VEGF therapy into the adjuvant setting in this respect has a strong rationale. VEGF is one of the first and most powerful pathways needed to build neovasculature. Adjuvant trial designs will investigate not only the duration of bevacizumab but also explore the combination with several chemotherapeutic agents.

Several promising future perspectives of antiangiogenic therapy need to be addressed. In the Her-2/neu overexpressing subgroup of breast cancer patients, the addition of bevacizumab to trastuzumab is studied in stage IV breast cancer. Furthermore, angiogenic ligands signal through receptor tyrosine kinases. By inhibiting these tyrosine kinases, downstream signaling occurring as a result of a multitude of proangiogenic ligands could be blocked. Currently, a multitude of small molecule inhibitors of receptor tyrosine kinases are under review in phase I/II studies.

\section{Self-Seeding of Cancer Cells}

Current models of cancer attribute the growth of cancer to the rapid proliferation of neoplastic cells in addition to low rates of apoptosis. The main reason for cancer progression locally along this train of thought is thus proliferation. Metastasis and cell invasion have been defined as cell movements fairly specific to cancerous cells and contribute to the initiation and progression of disease. Dr. Larry Norton (Memorial SloanKettering Cancer Center, New York, USA) reviewed an integrating concept with regard to the contribution of proliferation and cell movement to cancer progression [14]. Instead of simply attributing the growth of a tumor mass to proliferation, single cells could depart from the tumor, extravasate and gain access to the entire systemic circulation, and in a further step return to the site of origin. This pattern of self-seeding could induce a specific pattern of growth to both the primary tumor and metastasis. The tumor mass would then be rendered a dense collection of many small, adjoining fields of growth, rather than a more or less centrifugal growth pattern. Several lines of evidence ranging from genetic backgrounds, mathematical models of growth to molecular profiles were presented to undermine the hypothesis. Using this information as a biological background, it then becomes possible to imagine tumors with different phenotypes concerning their ability to proliferate or self-seed. In the case of a strong proliferative phenotype, typical cytotoxic therapy that targets mitosis would be sufficient. In most other cases, therapy should include strategies that may stop the process of growth via self-seeding. A current example would be antiangiogenetic agents.

\section{Conclusion}

Breast cancer biology may be fairly well understood from a multitude of backgrounds. Information stemming from different areas of research becomes complimentary to appreciating a fundamental biological understanding of breast cancer. For example, genetic profiling has identified subtypes of breast cancer that are now well compatible with many of our clinical concepts previously developed. Indeed, endocrine-related breast cancer that has been identified both by its hormone dependency to achieve growth and its endocrine receptors segregates closely in genetic clusters. Germline mutations in single genes such as BRCA1 lead to tumor phenotypes with high similarity to a subgroup of sporadic breast cancer that can be identified by a) immunohistochemistry or b) genetic profiling. The study of this data together may reveal the common etiologies of these neoplasms, and this understanding could be more complete than information stemming from just a single background. In conclusion, future therapeutic targets and agents depend on a fundamental biological appreciation of breast cancer. In order to elaborate the most comprehensive understanding, research networks will have to function on a broad scale and can then ultimately serve our patients. 


\section{References}

1 Curigliano G, Spitaleri G, Pietri E, Rescigno M, de Braud F, Cardillo A, Munzone E, Rocca A, Bonizzi G, Brichard V, Orlando L, Goldhirsch A: Breast cancer vaccines: a clinical reality or fairy tale? Ann Oncol 2006;17:750-762.

2 Dubsky $\mathrm{P}$, Ueno H, Piqueras B, Connolly J, Banchereau J, Palucka AK: Human dendritic cell subsets for vaccination. J Clin Immunol 2005;25: 551-572.

3 Hu Z, Fan C, Oh DS, Marron JS, He X, Qaqish BF, Livasy C, Carey LA, Reynolds E, Dressler L, Nobel A, Parker J, Ewend MG, Sawyer LR, Wu J, Liu Y, Nanda R, Tretiakova M, Ruiz Orrico A, Dreher D, Palazzo JP, Perreard L, Nelson E, Mone M, Hansen H, Mullins M, Quackenbush JF, Ellis MJ, Olopade OI, Bernard PS, Perou CM: The molecular portraits of breast tumors are conserved across microarray platforms. BMC Genomics 2006; 7:96.

4 Paik S, Shak S, Tang G, Kim C, Baker J, Cronin M, Baehner FL, Walker MG, Watson D, Park T, Hiller W, Fisher ER, Wickerham DL, Bryant J, Wolmark $\mathrm{N}$ : A multigene assay to predict recurrence of tamoxifen-treated, node-negative breast cancer. $\mathrm{N}$ Engl J Med 2004:351:2817-2826.

5 Schoppmann SF, Fenzl A, Nagy K, Unger S, Bayer G, Geleff S, Gnant M, Horvat R, Jakesz R, Birner P: VEGF-C expressing tumor-associated macrophages in lymph node positive breast cancer: impact on lymphangiogenesis and survival. Surgery 2006;139:839-846.

6 Mantovani A, Sica A, Locati M: New vistas on macrophage differentiation and activation. Eur J Immunol 2007:37:14-16.

7 Rhoden KJ, Unger K, Salvatore G, Yilmaz Y, Vovk V, Chiappetta G, Qumsiyeh MB, Rothstein JL, Fusco A, Santoro M, Zitzelsberger H, Tallini G: $\mathrm{RET} /$ papillary thyroid cancer rearrangement in nonneoplastic thyrocytes: follicular cells of Hashimoto's thyroiditis share low-level recombination events with a subset of papillary carcinoma. J Clin Endocrinol Metab 2006;91:2414-2423.

8 Walsh T, King MC: Ten genes for inherited breast cancer. Cancer Cell 2007;11:103-105.

$\checkmark 9$ Turner N, Tutt A, Ashworth A: Hallmarks of 'BRCAness' in sporadic cancers. Nat Rev Cancer 2004; 4:814-819.

10 Fan C, Oh DS, Wessels L, Weigelt B, Nuyten DS, Nobel AB, van't Veer LJ, Perou CM: Concordance among gene-expression-based predictors for breast cancer. N Engl J Med 2006;355:560-569.

$\checkmark 11$ Perou CM, Sorlie T, Eisen MB, van de Rijn M, Jeffrey SS, Rees CA, Pollack JR, Ross DT, Johnsen H, Akslen LA, Fluge O, Pergamenschikov A, Williams C, Zhu SX, Lonning PE, Borresen-Dale AL, Brown PO, Botstein D: Molecular portraits of human breast tumours. Nature 2000;406:747-752.

12 Ciana P, Scarlatti F, Biserni A, Ottobrini L, Brena A, Lana A, Zagari F, Lucignani G, Maggi A: The dynamics of estrogen receptor activity. Maturitas 2006;54:315-320.

13 Miller KD: E2100: a phase III trial of paclitaxel versus paclitaxel/bevacizumab for metastatic breast cancer. Clin Breast Cancer 2003;3:421-422.

14 Norton L, Massague J: Is cancer a disease of selfseeding? Nat Med 2006;12:875-878. 